\title{
Fluid Queue Models of Renewable Energy Storage
}

\author{
Gareth L. Jones, Peter G. Harrison, Uli Harder, Anthony J. Field \\ Imperial College London \\ 180 Queen's Gate, London, SW7 2RH \\ Email: \{gljones,uh,pgh,ajf\}@doc.ic.ac.uk
}

\begin{abstract}
In this extended abstract we introduce an approximation algorithm for the evaluation of networks of fluid queues. Such models can be used to describe the generation and storage of renewable energy.

We discuss how our algorithm would be applied to an example where the approximation performs very well, and note a modification to the model which would result in a poorer result.
\end{abstract}

\section{INTRODUCTION}

Single fluid queues have received attention in the literature and numerous results are known (see for example the surveys of Kulkarni [10] or Ahn and Ramaswami [3]). However, the analysis of a network of these queues is not an easy model to consider and few results exist for these networks in general. It has been shown that such networks do not exhibit the productform property held by many discrete queueing networks which allows for quick and efficient evaluation of stationary metrics [4], [9].

Recently the authors of this paper argued that fluid queue networks can describe the problem energy producers face in routing and allocating storing energy produced from renewable sources [8]. As renewable power becomes more a more prevelant source of generating capacity understanding how these networks work will become increasingly important. The Economist recently reported that the state of grid operator of Texas (which has recently invested heavily in wind power) found themselves being forced to buy electricity at time "an eye-watering 30 times the normal price" [2]. The state has plenty of capacity - nearly twice the level of total demandbut wind power generates most power at night, while Texan consumers use most power for air conditioning during hot summer afternoons.

In this paper we introduce a simple example (previously introduced in [8]) of an energy storage network and show how we can use an approximation algorithm to compute an approximate performance metric. We compare this to results from simulations and our results are initially encouraging. There is significant scope for both the model and approximation to be improved and we highlight some ideas at the end of the paper.

\section{FLUID QUEUES AND NETWORKS OF FLUID QUEUES}

\section{A. Fluid queues}

A fluid queue is a bivariate stochastic process $\left(J_{t}, X_{t}\right)$ with an associated input rate vector $\lambda$ and service rate scalar $\mu$. The process $J_{t}$ is a continuous time Markov chain on the states

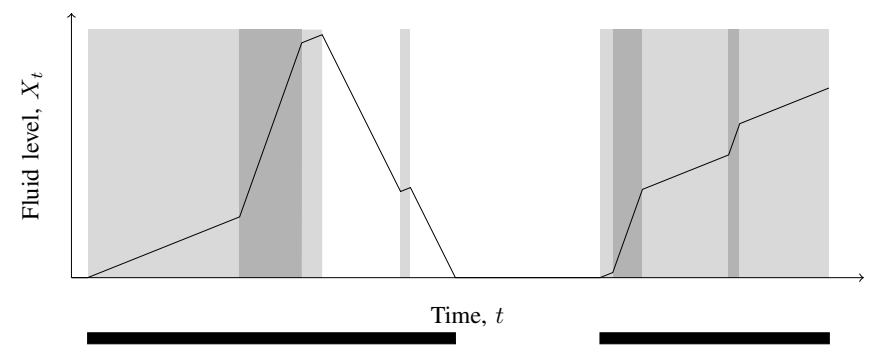

Fig. 1. Sample trace from the fluid level at node 1. The areas with a light grey highlight correspond to the times when one source was feeding the node and the areas with a dary grey highlight correspond to the times when both sources were feeding the node. The busy periods are highlighted with a thick black line. The first is a complete busy period, the second has not finished in the duration of this trace.

$\{1,2, \ldots, n\}$ and $\mathbf{r}=\lambda-\mu \cdot 1$ is an $n$-dimensional vector. $X_{t}$ is a stochastic process such that at time $t$, when $J_{t}=i$,

$$
\frac{\mathrm{d} X_{t}}{\mathrm{~d} t}= \begin{cases}r_{i} & \text { if } X_{t}>0 \\ \max \left(r_{i}, 0\right) & \text { if } X_{t}=0 .\end{cases}
$$

Fluid queues are a sub-class of piecewise deterministic Markov processes. A sample trace from a fluid queue with two inputs (node 1 in Figure 2) is shown in Figure 1. The output process of a fluid queue $Y_{t}$ is given by

$$
Y_{t}= \begin{cases}\mu & \text { if } X_{t}>0 \\ \lambda_{i} & \text { if } X_{t}=0\end{cases}
$$

Such models have been studied extensively in the literature. See [10] for stationary distribution results and [7] for computation of the busy period distribution. The busy period is the time period for which the fluid queue contains fluid (has positive fluid level).

Node 1 is a fluid queue with $\mu=25, \lambda=(60,30,30,0)$ and $Q$ matrix given by the Kronecker sum of the two source $Q$ matrices.

A sample trace at node 1 is shown in 1 where a busy period is highlighted with a bold line. The output process of node 1 has two states: 0 and 25 . The time spent in state 0 is exponential with rate parameter 2 . The time spent in state 25 is the busy period of the queue (highlighted with a bold line in the trace) and is not exponentially distributed.

\section{B. Fluid queиe networks}

In a fluid queue networks we consider a number of fluid queues linked together in a feedforward manner. We define 


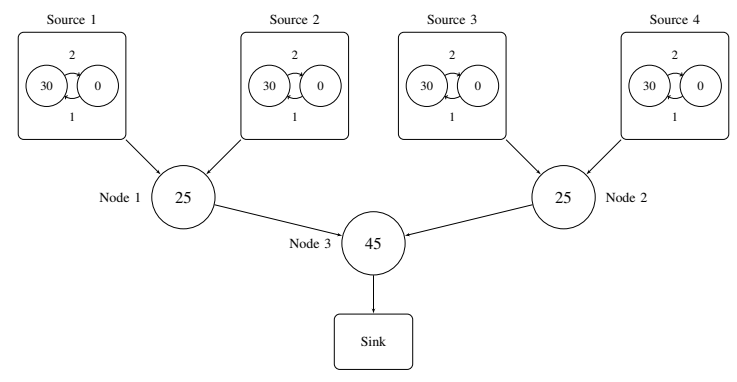

Fig. 2. The example network considered in this paper. Fluid is generated at each of the four sources at rate 30 .

a routing matrix $P$ with rows $i$ that describe the proportion of fluid leaving node $i$ that is sent to nodes $1,2, \ldots, n$. In the network considered here the routing matrix is particularly simple as all fluid leaving a source or node is routed to only one place. More generally we can apply the ideas here to networks where nodes can be numbered in such a way that $P$ is an upper diagonal matrix with zeros on the diagonal. We do not consider networks with loops/cycles.

\section{EXAMPLE}

\section{A. Model description}

We consider the example network in Figure 2. There are four independent on/off sources, each of which produces power for $1 / 3$ of the time on average. A $33 \%$ capacity factor is a reasonable approximation to that of a wind turbine (typically $20-40 \%$ [1]).

In Figure 2, nodes 1 and 2 represent storage facilities that smooth out the intermittent power produced by the four sources. Node 3 represents a customer who requires power at rate 45 . The four sources each have an on rate of 30 .

We compute an approximation for the moments of the busy period at node 3 . The first moment (the mean busy period) corresponds to the average length of time for which the customer at node 3 has their power demands satisfied.

\section{B. Approximation algorithm}

We start the algorithm with details of the output of the source nodes. We work through each node in the tree-like network approximating the output as a Markov modulated flow, using these approximations as inputs to approximate the behaviour at sebsequent nodes.

We start at nodes 1 and 2 using the method in Field and Harrison [5]. They note that the holding time of the off period in the output process from these nodes has the same distribution as the off period of the combined input process-exponentially distributed with parameter 2 . The on period of the output from nodes 1 and 2 is approximated by an exponential distribution with parameter chosen so as to conserve throughput.

The output of nodes 1 and 2 is approximated by a continuous time Markov chain with with three states: 25, 25, 0 and structure as in [5]. Then we compute metrics for an approximate node 3 fed by these two approximated inputs.
We use the recent Field and Harrison result [6] to compute the busy period at this node. We found both the approximate value and value from simulation to be 2.26 , so we see the approximation performs well.

(Due to complications in dealing with repeated eigenvalues, the approximations actually assumed the service rates were 24.9 and 25.1 at nodes 1 and 2.)

\section{FUTURE WORK}

In this paper we have applied a recent theoretical result of Field and Harrison to improve the applicability of an approximation algorithm previously proposed by the same authors. We have considered a simple example and seen good performance.

However, to extend this approximation method to a more general network there are issues that need to be carefully considered. The output from a general queueing node will have more than two states (not just on/off) and these smaller flow rates will need to be dealt with carefully, especially the parameters chosen to link them to the busy period rates.

Also, the network considered here was particularly simple as there were no dependencies between flows. Suppose we had taken sources 2 and 3 and combined them in to one on/off source of rate 60 , the output of which was split between nodes 1 and 2 equally. This would increase the mean busy period to 3.00 , while the approximation presented here (ignoring this dependence) would remain unchanged.

With regards to the application we need to parametrise a phase type input to describe real power sources and consider losses at queueing nodes due to transmission/storage costs. Finite buffers will also need to be considered, and the reaction of the network to a full buffer. In a network with static routing excess fluid will be lost and a buffer overflow, however some dynamic routing protocol could help conserve fluid.

\section{REFERENCES}

[1] Wind Power: Capacity Factor, Intermittency, and what happens when the wind doesn't blow? University of Massachusetts, 2009.

[2] Packing some power. The Economist, 3 March 2012 http://www. economist. com/node/21548495.

[3] S. Ahn and V. Ramaswami. Fluid Flow Models and Queues-A Connection by Stochastic Coupling. Stochastic Models, 19(3):325-348, Aug. 2003.

[4] A. J. Field and P. G. Harrison. An Approximate Compositional Approach to the Analysis of Fluid Queue Networks. Performance Evaluation, 64(9-12):1137-1152, October 2007.

[5] A. J. Field and P. G. Harrison. Approximate Analysis of a Network of Fluid Queues. In Workshop on Mathematical performance Modeling and Analysis (MAMA), June 2007, volume 35 of Performance Evaluation Review, pages 30-32, August 2007.

[6] T. Field. Jinqs: An extensible library for simulating multiclass queueing network. 2010.

[7] T. Field and P. G. Harrison. Busy periods in fluid queues with multiple emptying input states. Journal of Applied Probability, 47:474-497, 2010.

[8] G. Jones and P. G. Harrison. Using Fluid Queues to Model Energy Storage and Distribution. In Imperial College Energy and Performance Colloquium (ICEP), May 2012.

[9] O. Kella. Non-product form of two-dimensional fluid networks with dependent Lévy inputs. Journal of Applied Probability, 37(4):11171122, 2000.

[10] V. G. Kulkarni. Fluid models for single buffer systems. Frontiers in Queueing: Models and Applications in Science and Engineering, pages 321-338, 1997. 